\title{
Video Article \\ A Simple Method to Identify Kinases That Regulate Embryonic Stem Cell Pluripotency by High-throughput Inhibitor Screening
}

\author{
Charles A. C. Williams ${ }^{1}$, Nathanael S. Gray ${ }^{2,3}$, Greg M. Findlay ${ }^{1}$ \\ ${ }^{1}$ The MRC Protein Phosphorylation and Ubiquitylation Unit, School of Life Sciences, University of Dundee \\ ${ }^{2}$ Department of Cancer Biology, Dana-Farber Cancer Institute \\ ${ }^{3}$ Department of Biological Chemistry and Molecular Pharmacology, Harvard Medical School
}

Correspondence to: Greg M. Findlay at g.m.findlay@dundee.ac.uk

URL: https://www.jove.com/video/55515

DOI: doi: $10.3791 / 55515$

Keywords: Developmental Biology, Issue 123, Protein Kinase, Embryonic Stem Cell, Pluripotency, Naïve-Primed Transition, Kinase Inhibitors, HighThroughput Screen

Date Published: 5/12/2017

Citation: Williams, C.A., Gray, N.S., Findlay, G.M. A Simple Method to Identify Kinases That Regulate Embryonic Stem Cell Pluripotency by Highthroughput Inhibitor Screening. J. Vis. Exp. (123), e55515, doi:10.3791/55515 (2017).

\section{Abstract}

Embryonic stem cells (ESCs) can self-renew or differentiate into all cell types, a phenomenon known as pluripotency. Distinct pluripotent states have been described, termed "naïve" and "primed" pluripotency. The mechanisms that control naïve-primed transition are poorly understood. In particular, we remain poorly informed about protein kinases that specify naïve and primed pluripotent states, despite increasing availability of high-quality tool compounds to probe kinase function. Here, we describe a scalable platform to perform targeted small molecule screens for kinase regulators of the naïve-primed pluripotent transition in mouse ESCs. This approach utilizes simple cell culture conditions and standard reagents, materials and equipment to uncover and validate kinase inhibitors with hitherto unappreciated effects on pluripotency. We discuss potential applications for this technology, including screening of other small molecule collections such as increasingly sophisticated kinase inhibitors and emerging libraries of epigenetic tool compounds.

\section{Video Link}

The video component of this article can be found at https://www.jove.com/video/55515/

\section{Introduction}

Embryonic stem cells (ESCs) have the capacity to self-renew or differentiate into any cell type in the adult body, a phenomenon known as pluripotency ${ }^{1}$. Recent evidence indicates that developmentally distinct pluripotent states exist, termed "naïve" and "primed" pluripotency ${ }^{2}$. Naïve ESCs represent a state of development similar to that found in the preimplantation embryo ${ }^{3}$. In contrast, primed pluripotent ESCs are poised to exit pluripotency and differentiate into specialized embryonic lineages ${ }^{4,5}$.

Naïve and primed pluripotent states are marked by distinct gene regulatory networks. Naïve pluripotency is characterized by expression of key pluripotency transcription factors such as Nanog, Krueppel-like transcription factors (Klfs), Rex $1^{2}$ and Esrrb ${ }^{6}$. In mouse ESCs (mESCs), primed pluripotency is characterized by reduced expression of naïve markers and a specific gene expression signature which includes the de novo DNA methyltransferase Dnmt $3 b^{7}$. In vivo, primed pluripotent post implantation epiblast stem cells (EpiSCs) ${ }^{4,5}$ additionally express the Epiblast marker Fgf5 and markers of lineage priming such as Brachyury ${ }^{8}$.

mESCs provide a tractable model to probe mechanisms that control naïve-primed pluripotent transitions in vitro. When cultured in leukemia inhibitory factor (LIF) and fetal bovine serum (FBS), mESCs undergo dynamic transition between naïve and primed pluripotent states ${ }^{9,10}$. LIFJak-Stat3 signaling functions to promote a naïve gene regulatory network ${ }^{11}$, whilst autocrine signaling via the fibroblast growth factor 4 (Fgf4) Erk $1 / 2$ pathway drives transition to the primed state ${ }^{12}$. However, it remains a challenge to systematically evaluate the role of protein kinases in specifying distinct pluripotent states.

Here, we describe a quantitative and scalable platform by which to perform targeted small molecule screens for kinase regulators of the naïveprimed pluripotent transition. We use simple mESC culture conditions and standard reagents, materials and equipment to uncover and validate kinase inhibitors with hitherto unappreciated ability to stabilize naïve pluripotency. Furthermore, we discuss potential extended applications for this technology, including for screening of other small molecule collections such as emerging inhibitor libraries targeting epigenetic regulators. 


\section{Collation of Small Molecule Kinase Inhibitor Libraries}

1. Collate inhibitor libraries from publicly accessible kinase inhibitor collections, e.g. Library of Integrated Network-based Cellular Signatures (LINCS) from Harvard Medical School, a targeted collection of 228 potent and selective kinase inhibitors (http://lincs.hms.harvard.edu), and the Protein Kinase Inhibitor Set (PKIS), a 376 compound collection assembled by industrial researchers (https://www.ebi.ac.uk/chembldb/ extra/PKIS) ${ }^{13}$. Alternatively, assemble a bespoke kinase inhibitor collection from commercially available compounds.

NOTE: In our laboratory, we have assembled a collection of 72 potent and selective tool kinase inhibitors covering 51 primary target kinases, all of which are available from commercial sources.

2. Assemble kinase inhibitors into 96-well plates for optimal high-throughput processing at multiple concentrations (use $1,0.1$ and $0.01 \mathrm{mM}$ ). Include in each plate control wells containing DMSO only and reference control inhibitors PD173074 (FGFRi) and Ruxolitinib (JAKi), which are known to promote naïve and primed pluripotent states respectively. Annotate in a spreadsheet or similar software.

\section{2. mESC Culture Conditions and Procedures for Screen Preparation}

1. Prepare $0.1 \%$ gelatin-coated $10 \mathrm{~cm}$ dishes by adding $5 \mathrm{~mL}$ of $0.1 \%(\mathrm{w} / \mathrm{v})$ gelatin to $10 \mathrm{~cm}$ plates and incubate at room temperature for $5 \mathrm{~min}$. Aspirate gelatin and let plate dry for $2 \mathrm{~min}$.

2. Culture any standard $\mathrm{mESC}$ line at $37^{\circ} \mathrm{C} 5 \% \mathrm{CO}_{2}$ on $0.1 \%$ gelatin-coated $10 \mathrm{~cm}$ dishes in standard mESC media (see Materials Table) containing $100 \mathrm{ng} / \mathrm{mL}$ GST-LIF, 10\% Fetal Calf Serum and 5\% Knockout Serum Replacement. Replace media every day and passage $\mathrm{mESC}$ at around $80 \%$ confluency every second day at a dilution of 1:5-1:10.

3. To passage $\mathrm{mESCs}$, aspirate media and wash with $5 \mathrm{~mL}$ of phosphate-buffered saline (PBS) per plate.

4. Add $1 \mathrm{~mL}$ trypsin-EDTA $\left(0.05 \%\right.$ Trypsin, $0.02 \%$ EDTA) per plate of $\mathrm{mESCs}$ and incubate at $37^{\circ} \mathrm{C}$ for $10 \mathrm{~min}$.

5. Resuspend trypsinized cells in $4 \mathrm{~mL}$ of $\mathrm{mESC}$ media and centrifuge at $1,200 \mathrm{rpm}$ for $5 \mathrm{~min}$.

6. Thoroughly resuspend cell pellet in $5 \mathrm{~mL}$ of $\mathrm{mESC}$ media, pipetting up and down to generate a single cell suspension.

7. Count cells by combining a $10 \mu \mathrm{L}$ cell suspension and $10 \mu \mathrm{L}$ of Trypan Blue $(0.4 \%)$. Place into a cell counting chamber or use a hemocytometer and light microscope.

8. Seed $3 \times 10^{3} \mathrm{mESC}$ into $0.1 \%$ gelatin coated 96 well plates, final volume $100 \mu \mathrm{L}$ of media, using a multichannel pipette.

9. Apply kinase inhibitors at 1:100 dilution ( $1 \mu \mathrm{L}$ inhibitor added to $100 \mu \mathrm{L}$ media) using a multi-channel pipette. Gently pipette media to mix inhibitor and cell suspension, then allow cells to settle in a tissue culture hood for $1 \mathrm{~h}$ to ensure equal distribution across the plating surface. Culture cells for $48 \mathrm{~h}$ without changing the media.

NOTE: Stock plates of 1/0.1/0.01 mM will give a final inhibitor concentration of 10/1/0.1 $\mu \mathrm{M}$ respectively. we recommend starting the screen at a final concentration of $1 \mu \mathrm{M}$ to ensure effective inhibition of primary target kinases whilst minimizing off-target effects.

\section{Kinase Inhibitor Screening Analysis}

1. Wash 96 well mESC plates in $200 \mu \mathrm{L}$ PBS using multi-channel aspirator and pipettes.

2. Make cell extracts in $150 \mu \mathrm{L}$ of lysis buffer (20 mM Tris pH 7.4, $150 \mathrm{mM} \mathrm{NaCl}, 1 \mathrm{mM}$ EDTA, $1 \%$ NP-40 (v/v), $0.5 \%$ sodium deoxycholate (w/v), $10 \mathrm{mM} \beta$-glycerophosphate, $10 \mathrm{mM}$ sodium pyrophosphate, $1 \mathrm{mM} \mathrm{NaF}, 2 \mathrm{mM} \mathrm{Na}_{3} \mathrm{VO}_{4}$, Complete Protease Inhibitor Cocktail Tablets).

3. Clarify extracts by centrifugation at $1,500 \times \mathrm{g}$ for $30 \mathrm{~min}$ in V-bottomed 96 -well plates.

4. Immobilize $100 \mu \mathrm{L}$ of supernatants onto a nitrocellulose membrane using a 96 -well vacuum dot blot manifold.

5. Dry the membrane, and stain with $40 \mathrm{~mL}$ of Ponceau $\mathrm{S}$ to ensure consistent transfer.

6. Wash membrane with TBST and block in TBST/3\% (w/v) milk.

7. Incubate in Nanog and Dnmt3b antibodies at a dilution of $1: 1,000(\mathrm{v} / \mathrm{v})$ in TBST/3\% (w/v) milk powder overnight.

8. Wash membranes in $3 \times 10 \mathrm{~min}$ in TBST and incubate in $30 \mathrm{~mL}$ of secondary antibodies at a dilution of 1:10,000 (v/v) in TBST/3\% (w/v) milk.

9. Develop using a digital immunoblotting imaging system.

NOTE: If possible, use quantitative fluorescence immunoblotting to detect Nanog and Dnmt3b. However, the background signal for Dnmt3b by fluorescence immunoblotting is too high. Therefore, $800 \mathrm{~nm}$ fluorescence anti-rabbit (Nanog) and anti-mouse-HRP (Dnmt3b) secondary antibodies are used.

\section{Analysis of Screen Data and Validation of Inhibitors as Bona Fide Pluripotency Regulators}

NOTE: This is a critical step to ensure that only bona fide pluripotency modulators are identified. It is essential to triage inhibitors based on both Nanog:Dnmt3b ratio and overall signal, to ensure that kinase inhibitors which adversely affect mESC survival are not selected for further analysis.

1. Quantify Nanog and Dnmt3b signal using immunoblotting analysis software, and import values into a spreadsheet or similar software. Calculate Nanog:Dnmt3b ratio for all samples compared to DMSO control. Also, determine total Nanog and Dnmt3b signal to ensure that kinase inhibitors do not affect Nanog:Dnmt3b ratio by altering mESC viability.

2. Use ranked Nanog:Dnmt3b ratios to identify inhibitors which promote naïve pluripotency (Nanog:Dnmt3b ratio higher than control) and primed pluripotency (Nanog:Dnmt3b ratio lower than control). Set cut-off at $2 x$ deviation from control values, and filter out kinase inhibitors which produce a total Nanog+Dnmt3b signal of less that $50 \%$ of the DMSO control value to generate a high confidence list of kinase inhibitors which stabilise naïve (high Nanog:Dnmt3b ratio) and primed (low Nanog:Dnmt3b ratio) pluripotent states. 
3. Validate kinase inhibitors by seeding mESCs at $2 \times 10^{5}$ cells per 6 well in $2 \mathrm{~mL}$ media, and add kinase inhibitors at $1 \mu \mathrm{M}$ for $48 \mathrm{~h}$, without changing media.

4. Lyse mESCs in lysis buffer and analyze Nanog and Dnmt3b expression by conventional SDS-PAGE immunoblotting.

5. Further validate hits by SDS-PAGE immunoblotting for naïve pluripotency markers KIf2 and Klf4, and Oct4 to ensure that pluripotency is maintained following $48 \mathrm{~h}$ inhibitor treatment.

\section{Representative Results}

Using the procedure presented here (Figure 1), we screen the LINCS library of 228 potent and selective kinase inhibitors to identify those which modulate $\mathrm{mESC}$ pluripotency. The library is prepared at a concentration of $0.1 \mathrm{mM}$ for a 1:100 dilution and final screening concentration of 1 $\mu \mathrm{M}$ in mESCs. $48 \mathrm{~h}$ later, mESCs were lysed and extracts prepared for quantitative dot blot analysis of Nanog and Dnmt3b expression (Figure 2, top). Results from other screening concentrations are not represented here for brevity. Nanog:Dnmt3b ratio is determined for each kinase inhibitor and compounds ranked and a 2x cut-off applied (Figure 2, bottom). Total Nanog and Dnmt3b signal relative to control is overlaid onto inhibitor ranking and subjected to a $0.5 x$ cut-off, to allow triaging of inhibitors which show significant mESC toxicity. Select kinase inhibitors which stabilize the naïve state are validated using conventional Nanog/Dnmt3b immunoblotting (Figure 3). The primary kinase targets of these inhibitors are presented in Table 1. We also demonstrate the applicability of this screen to identify inhibitors which stabilize the primed state ${ }^{14}$.
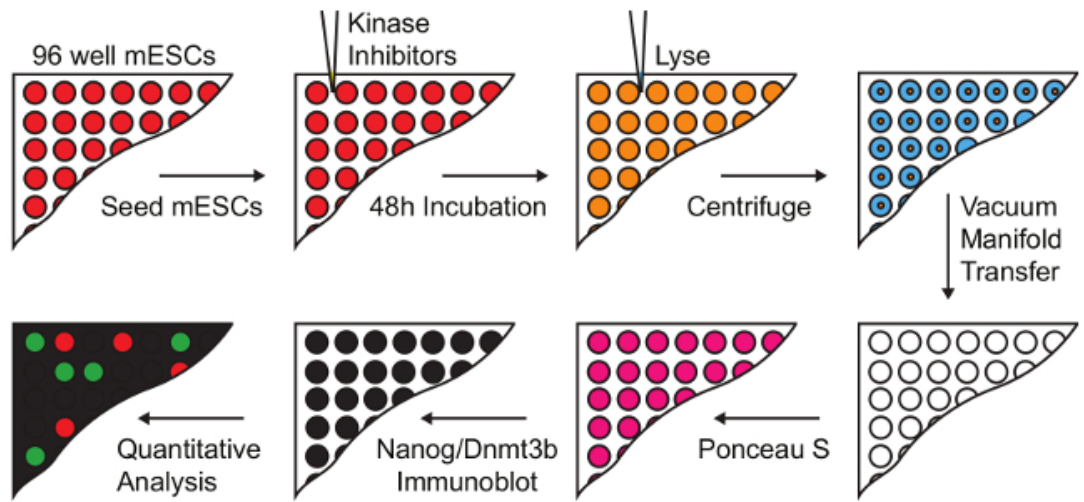

Figure 1: Workflow for screening kinase inhibitors that modulate naïve-primed transition. mESC were treated with a 228 compound kinase inhibitor library at a final concentration of $1 \mu \mathrm{M}$. Lysates were prepared and transferred onto nitrocellulose membranes for immuno dotblot analysis, and Nanog and Dnmt3b levels determined (Figure adapted from Williams et al. ${ }^{14}$ ). Please click here to view a larger version of this figure. 


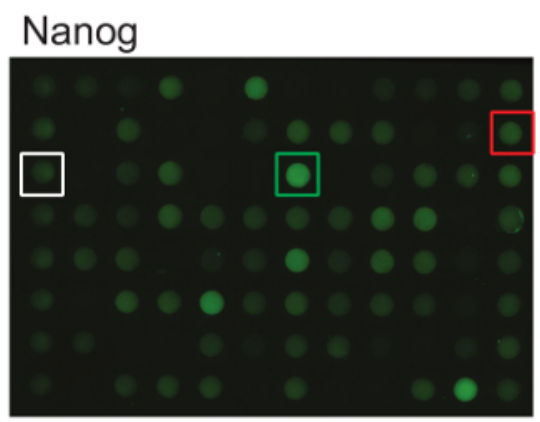

Dnmt3b $\square$ Ruxolitinib

PD173074 口DMSO

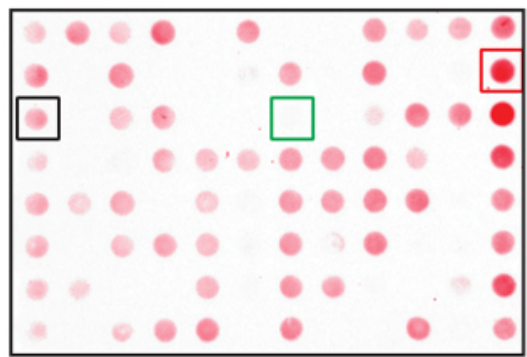

False Colour

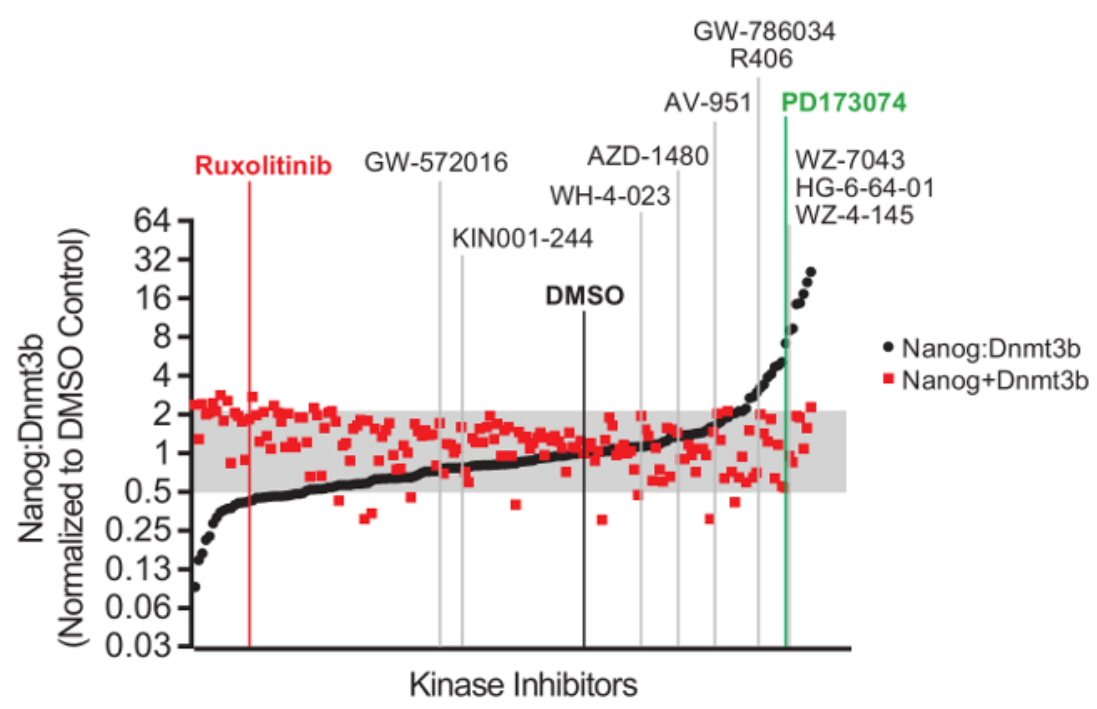

Figure 2: Naïve-primed pluripotency screen analysis and inhibitor identification. (Top) Representative Nanog and Dnmt3b immuno dotblot images. (Bottom) Nanog and Dnmt3b values for each kinase inhibitor were determined relative to the DMSO control, and Nanog:Dnmt3b ratios and total relative Nanog+Dnmt3b signal determined and inhibitors ranked in comparison to DMSO control. Inhibitors found to alter Nanog:Dnmt3b ratio beyond a 2-fold threshold above or below DMSO control were identified as drivers of naïve or primed pluripotency. A threshold of $2 x$ below DMSO control was set to triage inhibitors which compromise mESC survival. Inhibitors selected for further validation are highlighted. (Figure adapted from Williams et al. ${ }^{14}$ ). Please click here to view a larger version of this figure. 


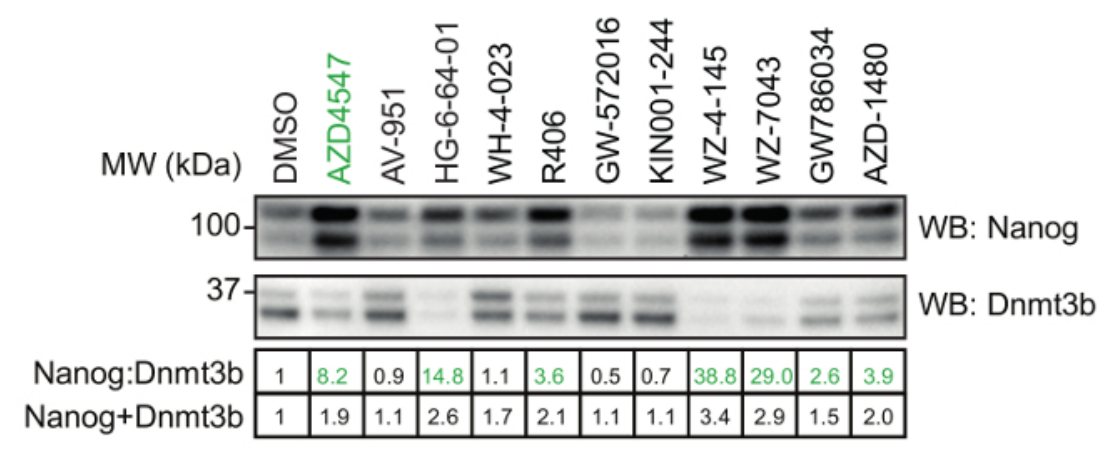

Figure 3: Validation of selected hit compounds identified. mESCs were treated with either $1 \mu$ M AZD4547 (a selective FGFR inhibitor) or the indicated inhibitors identified from the screen in Figure 2. Nanog:Dnmt3b levels determined by standard SDS-PAGE and immunoblot analysis. Numerical values of relative Nanog:Dnmt3b and Nanog+Dnmt3b are indicated in the table below, and inhibitors which stabilize the naïve pluripotent state highlighted in green. Please click here to view a larger version of this figure.

\begin{tabular}{|l|l|l|}
\hline Compound & Primary Targets & Other Targets \\
\hline AV-951 & VEGFR & \\
\hline HG-6-64-01 & $\begin{array}{l}\text { ABL, BRAF, RET, CSF1R, EGFR, EPHA8, FGFR, FLT3, KIT, LOK, } \\
\text { MAP4K1, p38b, MUSK,PDGFR, TAOK3, TNNI3K }\end{array}$ & \\
\hline WH-4-023 & Lck & Src \\
\hline R406 & Syk & EGFR \\
\hline GW-572016 & HER2 & \\
\hline KIN001-244 & PDK1 & \\
\hline WZ-4-145 & CSF1R, DDR1, EGFR, TIE1, PDGFR2 & \\
\hline WZ-7043 & CSF1R, DDR1, FGFR and TAO1 & VEGFR2, VEGFR3 \\
\hline GW786034 & VEGFR1 & \\
\hline AZD-1480 & JAK2 & \\
\hline
\end{tabular}

Table 1: Selected hit compounds and their primary kinase target(s).

\section{Discussion}

Here we demonstrate a widely accessible methodology to probe the role of kinase signalling pathways in regulating naïve-primed pluripotent transition. This addresses a key question in the ESC field. Although high-throughput genomics and transcriptomics approaches are routinely used to identify key transcriptional regulators of naïve and primed pluripotent states, elucidating pluripotency signalling networks has proven to be challenging. We now provide a flexible strategy employing chemical inhibitors to identify kinases that control naïve-primed pluripotent transition in mESCs. This relies on simple equipment, reagents and materials that are available to most laboratories that study cell signalling and ESC biology. Critical to the success of this platform is our development of a robust assay to quantify the transition between naïve and primed pluripotent states. Establishing this assay required significant iterative modifications to cell plating density, time of inhibitor incubation and immunoblotting conditions.

Small molecule approaches are gaining traction for rational use in therapeutic ESC applications ${ }^{15,16}$. A major strength of small molecule screening is that tool compounds are designed and/or modified for efficient cellular uptake. Transfection efficiency is not limiting, and use of hazardous delivery systems such as lentivirus is not necessary. Furthermore, inhibitors frequently inhibit multiple isoforms within a kinase family (e.g. Fgfr1-4, Jak1-3), which overcomes functional redundancy that hinders genetic/genomic interference techniques such as RNAi and CRISPR/ Cas9. As more potent and selective tool compounds become available from both academic and pharmaceutical drug discovery programmes, understudied and poorly understood kinases will be pushed to the forefront of ESC research. We therefore propose that this high-throughput screening approach will open up new avenues of research into core ESC regulatory networks.

One minor limitation of this technology is that even the most potent and selective small molecule inhibitors engage and inhibit multiple unrelated kinases in vivo. However, increasingly comprehensive kinase inhibitor profiling data allows functional targets of kinase inhibitors to be readily identified (http://lincs.hms.harvard.edu/kinomescan; http://www.kinase-screen.mrc.ac.uk/kinase-inhibitors). Finally, in principle our platform can be applied to interrogate any small molecule collection and/or cellular assay for which high-quality immunoblotting antibodies are available. This will allow study of multiple regulatory systems in pluripotency regulation and facilitate identification of small molecule inhibitors which modify diverse cellular processes. Specifically, we envisage that application of emerging small molecule collections such as the epigenetic probes being 
developed by the Structural Genomics Consortium (http://www.thesgc.org/chemical-probes/epigenetics) has the potential to uncover further novel regulators of pluripotent transitions.

\section{Disclosures}

The authors declare no conflicts of interest arising from this study.

\section{Acknowledgements}

C.A.C.W is supported by a Medical Research Council PhD studentship. G.M.F. is supported in part by a Medical Research Council New Investigator Award (MR/N000609/1) and a Tenovus Scotland research grant.

\section{References}

1. Evans, M. J., \& Kaufman, M. H. Establishment in culture of pluripotential cells from mouse embryos. Nature. 292 (5819), 154-156 (1981).

2. Nichols, J., \& Smith, A. Naive and primed pluripotent states. Cell Stem Cell. 4 (6), 487-92 (2009).

3. Boroviak, T., et al. Lineage-Specific Profiling Delineates the Emergence and Progression of Naive Pluripotency in Mammalian Embryogenesis. Developmental Cell. 35 (3), 366-382 (2015).

4. Tesar, P. J., et al. New cell lines from mouse epiblast share defining features with human embryonic stem cells. Nature. 448 (7150), 196-199 (2007).

5. Brons, I. G. M., et al. Derivation of pluripotent epiblast stem cells from mammalian embryos. Nature. 448 (7150), 191-195 (2007).

6. Festuccia, N., et al. Esrrb is a direct Nanog target gene that can substitute for Nanog function in pluripotent cells. Cell Stem Cell. 11 (4), 477-490 (2012).

7. Ficz, G., et al. FGF signaling inhibition in ESCs drives rapid genome-wide demethylation to the epigenetic ground state of pluripotency. Cell Stem Cell. 13 (3), 351-359 (2013).

8. Guo, G., et al. Klf4 reverts developmentally programmed restriction of ground state pluripotency. Development. 136 (7), 1063-9 (2009).

9. Chambers, I., et al. Nanog safeguards pluripotency and mediates germline development. Nature. 450 (7173), 1230-1234 (2007).

10. Findlay, G. M., et al. Interaction domains of Sos1/Grb2 are finely tuned for cooperative control of embryonic stem cell fate. Cell. 152 (5), 1008-1020 (2013).

11. Niwa, H., Burdon, T., Chambers, I., \& Smith, A. Self-renewal of pluripotent embryonic stem cells is mediated via activation of STAT3. Genes Dev . 12 (13), 2048-60 (1998).

12. Kunath, T., et al. FGF stimulation of the Erk1/2 signalling cascade triggers transition of pluripotent embryonic stem cells from self-renewal to lineage commitment. Development. 134 (16), 2895-902 (2007).

13. Elkins, J. M., et al. Comprehensive characterization of the Published Kinase Inhibitor Set. Nature Biotechnology. 34 (1), $95-103$ (2016).

14. Williams, C. A. C., et al. Erk5 Is a Key Regulator of Naive-Primed Transition and Embryonic Stem Cell Identity. Cell Reports. 16 (7), $1820-8$ (2016).

15. Cao, N., et al. Conversion of human fibroblasts into functional cardiomyocytes by small molecules. Science. 352 (6290), aaf1502 (2016).

16. Zhang, M., et al. Pharmacological reprogramming of fibroblasts into neural stem cells by signaling-directed transcriptional activation. Cell Stem Cell. 18 (5), 653-667 (2016). 